\title{
Audit analysing the outcome of patients referred to the rapid-access chest pain clinic and assessing the positive predictive value of the rapid-access chest pain clinic of patients with coronary artery disease
}

\author{
Authors: Khizar Khan-Mahmood, Viraj Patel and Mark Scoote
}

\section{Aims}

Analyse outcomes of patients referred to the rapid-access chest pain clinic (RACPC). Identifying positive predictive rate of angiograms, highlighting patterns of referral and to examine clinical outcomes of attendants, assessing efficiency.

\section{Methods}

1,212 patients were sampled. Inclusion criteria: all referrals to RACPC between 1 February 2014 and 31 January 2015. Demographic, referral source and management outcomes were analysed. The subset of patients who were referred to have angiograms was further analysed to assess their outcome.

\section{Results}

Mean age: 61, female patients: 618, male patients: 594 . 81.4\% of referrals were from GPs. $49.3 \%$ of referrals were discharged with no change in management. $40.9 \%$ of referrals underwent further investigations, of which $28 \%$ were angiograms. Of all patients who underwent angiograms, $45.6 \%$ of patients required intervention. Positive predictive value of RACPC, referring patients for angiogram who had coronary artery disease (CAD) was $74.4 \%$.

\section{Conclusions}

The majority of referrals were from GPs and almost half required no intervention, questioning whether referrals were appropriate. As clinics are already overbooked, are there changes that can be made to reduce inappropriate referrals from primary care, eg consultant telephone advice provision? Audit confirms that RACPCs are an effective and efficient tool to assess patients with angina, and are good at highlighting patients with CAD requiring intervention. RACPCs are an effective nurse-led outpatient service.

\section{Conflict of interest statement}

No conflict of interest identified. 